\title{
A Note on the Covariant Anomaly as an Equivariant Momentum Mapping
}

\author{
David Bao ${ }^{1, \star}$ and V. P. Nair ${ }^{2, \star \star}$ \\ 1 School of Mathematics, The Institute for Advanced Study, Princeton, NJ 08540, USA and \\ Department of Mathematics, University of Houston-University Park, Houston, TX77004, USA \\ 2 School of Natural Sciences, The Institute for Advanced Study, Princeton, NJ 08540, USA
}

\begin{abstract}
We show that there is a natural gauge invariant presymplectic structure $\omega$ on the space $\mathscr{A}$ of all vector potentials. The covariant axial anomaly $\tilde{G}$ is found to be the essentially unique infinitesimally equivariant momentum mapping for the action of the group of gauge transformations on $(\mathscr{A}, \omega)$. The infinitesimal equivariance of $\widetilde{G}$ is shown to be equivalent to the Wess-Zumino consistency condition for the consistent axial anomaly $G$. We also show that the $X$ operator of Bardeen and Zumino, which relates $G$ and $\tilde{G}$, corresponds to the one-form (on $\mathscr{A}$ ) of the presymplectic structure $\omega$.
\end{abstract}

\section{Introduction}

The mathematical structure of the consistent axial anomaly $G$ can be studied from several viewpoints. For example, one can use differential geometric and algebraic techniques on spacetime, as in Zumino [16] and Zumino et al. [17]; or one can use differential geometry and elliptic analysis directly on the space $\mathscr{A}$ of all connections (vector potentials), as done by Atiyah and Singer [2]. An important ingredient about $G$ is its integrability criterion, the Wess-Zumino consistency condition. To go from $G$ to the covariant axial anomaly $\tilde{G}$, one can use the explicitly given $X$ operator of Bardeen and Zumino [4].

The present note is motivated by two questions: What is the intrinsic integrability condition for the covariant anomaly $\widetilde{G}$ ? And what is the geometrical interpretation of the aforementioned $X$ operator? Inspired by Atiyah and Singer's success in dealing directly with the geometry of the space $\mathscr{A}$ of all connections, we feel it would be instructive to examine our questions from the viewpoint of presymplectic geometry on $\mathscr{A}$. The abstract summarizes our results.

Our presentation is organized as follows. Section 1 sets up the terminology and notation concerning $\mathscr{A}$ and the group of gauge transformations which acts on it,

* Research supported in part by NSF grant MCS 81-08814(A03)

$\star \star$ Research supported by the U.S. Department of Energy under contract number DE AC02 76ER 02220 
and records some facts about symmetric products of Lie algebra valued differential forms. Section 2 briefly reviews axial anomalies. The main results are derived in Sect. 3, and discussions are contained in Sect. 4. Our objective of making this work readable for both mathematicians and physicists has contributed to its length.

\section{Connections, Gauge Transformations, and Symmetric Products}

Let $G$ be a Lie group in a matrix representation, and let $g$ be its Lie algebra. The adjoint action of $G$ on $g$ is $\operatorname{Ad}_{g} \lambda=g \lambda g^{-1}$, whose derivative at the identity gives the matrix bracket $\left[\lambda^{\prime}, \lambda\right]=\lambda^{\prime} \lambda-\lambda \lambda^{\prime}$.

Let $P \stackrel{\pi}{\longrightarrow} M$ be a principal bundle over spacetime $M$, with structure group $G$ whose action on $P$ is on the right $\left((p \cdot g) \cdot g^{\prime}=p \cdot\left(g g^{\prime}\right)\right)$, free (that is, without fixed points), and transitive on each fiber $\left[\pi(p)=\pi\left(p^{\prime}\right) \Rightarrow p \cdot g=p^{\prime}\right.$ for some $\left.g\right]$. Let $(\cdot g)$ denote the diffeomorphism $p \mapsto p \cdot g$. A vector $V$ is vertical if it is tangent to some fiber (that is, $\pi_{*} V=0$ ). The action is described infinitesimally by the "rigid" vertical vector fields $\bar{\lambda}$, where

$$
\bar{\lambda}(p):=\left.\frac{d}{d t}\right|_{t=0} p \cdot(\exp t \lambda), \quad \lambda \in \mathfrak{g} .
$$

The $\bar{\lambda}$ 's are nowhere zero (if $\lambda \neq 0$ ) because the action is free; they span the vertical subspace at each point. The map $\lambda \mapsto \bar{\lambda}$ obeys an equivariance whose finite and infinitesimal versions are respectively [5]

$$
\overline{\operatorname{Ad}_{g}^{-1} \lambda}=(\cdot g)_{*} \bar{\lambda}, \quad \overline{\left[\lambda, \lambda^{\prime}\right]}=\left[\bar{\lambda}, \overline{\lambda^{\prime}}\right]:=\mathscr{L}_{\bar{\lambda}} \overline{\lambda^{\prime}} .
$$

$\mathrm{A} g$-valued differential form $\Omega$ on $P$ is equivariant if $\operatorname{Ad}_{g}^{-1} \circ \Omega=(\cdot g)^{*} \Omega$, and is said to be horizontal if it vanishes whenever one of its arguments is vertical.

Let $\mathscr{A}$ denote the space of connections of the principal bundle. Each $A \in \mathscr{A}$ is an equivariant $\mathrm{g}$-valued one-form on $P$ such that

$$
A(\bar{\lambda})=\lambda \text {. }
$$

Thus there is no zero connection. $\mathscr{A}$ is affine: each tangent vector $\tau$ at $A$ is of the form $\pm\left(A^{\prime}-A\right)$ for some $A^{\prime} \in \mathscr{A}$, hence is a $\mathfrak{g}$-valued one-form on $P$ which is equivariant and horizontal [due to Eq. (1.3)]. By equivariance, no information is lost by choosing local sections $s: U \subseteq M \rightarrow P$ and working with $A^{s}:=s^{*} A$ and $\tau^{s}:=s^{*} \tau$ which, under a change of sections $s^{\prime}(x)=s(x) \cdot g(x)$, transform like $A^{s^{\prime}}$ $=g^{-1} A^{s} g+g^{-1} d g$ and $\tau^{s^{\prime}}=g^{-1} \tau^{s} g$. We suppress the superscript $s$ whenever possible.

Let $H_{A}$ denote "horizontal" projection onto the null space of $A$, which complements the vertical subspace at each point. $D_{A} \Omega:=(d \Omega) \circ H_{A}$ is horizontal and defines the exterior covariant derivative of forms on $P$. The horizontal 2-form $F_{A}:=D_{A} A$ is the curvature of $A$. The structural equations [5] say that $F_{A}=d A$ $+A^{2}$ and, for any horizontal equivariant $g$-valued $r$-form $\Omega$ on $P, D_{A} \Omega=d \Omega+A \Omega$ $-(-1)^{r} \Omega A$. This latter formula does not apply to the non-horizontal $A$, but it does apply to $F_{A}$ and yields the Bianchi identity $D_{A} F_{A}=0$.

Computations on $M$ are simplified by using, for each $B \in \mathscr{B}:=\{$ locally defined $\mathrm{g}$-valued one-forms on $M\}$, the abbreviations $F_{B}:=d B+B^{2}$ and $D_{B} \zeta:=d \zeta+B \zeta$ 
$-(-1)^{r} \zeta B$ for $\zeta \in \Lambda^{r}(M) \otimes \mathfrak{g}$. [For example, $F_{t A}=t d A+t^{2} A^{2}=t F_{A}+\left(t^{2}-t\right) A^{2}$ and $D_{t A} t A=t d A+2 t^{2} A^{2}$, where $A$ means $s^{*} A$.] The generalized Bianchi identity $D_{B} F_{B}=0$, equivalently $D_{B} F_{B}^{m}=0 \forall m$, then follows. $\mathscr{B}$ may be identified with the linear space of equivariant $\mathfrak{g}$-valued one-forms on $P$, in which case it properly contains $\mathscr{A}$, since $t A(0 \leqq t \leqq 1, A \in \mathscr{A})$ is a ray in $\mathscr{B}$ which meets $\mathscr{A}$ only when $t=1$.

Let $\mathscr{G}$ be the group of gauge transformations of $P$. Each $\varphi \in \mathscr{G}$ is a diffeomorphism on $P$ which projects to the identity map on $M$, and is equivariant: $\varphi(p \cdot g)=(\varphi p) \cdot g$. Its Lie algebra Lie $\mathscr{G}$ consists of all vertical vector fields $V$ on $P$ which obey the equivariance $V(p \cdot g)=(\cdot g)_{*}(V p)$. Through local sections $s, \varphi$ and $V$ are described by maps $\gamma: U \subseteq M \rightarrow G$ and $\Lambda: U \subseteq M \rightarrow \mathfrak{g}$, where

$$
\varphi(s(x))=s(x) \cdot \gamma(x), \quad V(s(x))=\overline{\Lambda(x)}(s(x)) .
$$

Note that $\varphi^{-1}(s(x))=s(x) \cdot \gamma^{-1}(x)$, since the action is free. And $\Lambda(x)=A(V(s x))$ for any connection $A$. The equivariance of $V$ and Eq. (1.2) give, under a change of section,

$$
\Lambda^{s^{\prime}}=g^{-1} \Lambda^{s} g
$$

The adjoint action of $\mathscr{G}$ on $\operatorname{Lie} \mathscr{G}$ is

$$
\operatorname{Ad}_{\varphi} V=\varphi_{*} V \leftrightarrow \gamma \Lambda \gamma^{-1} .
$$

Note that $\left.\operatorname{(Ad}_{\varphi} V\right)(p)=\left.\frac{d}{d t}\right|_{t=0}(\varphi \circ \exp t V)\left(\varphi^{-1} p\right)=\varphi_{*}\left(V\left(\varphi^{-1} p\right)\right)$ is clearly vertical, and is equivariant since $\varphi$ and $V$ are. The local description follows from $\varphi^{-1}(s(x))=s(x) \cdot \gamma^{-1}(x)$, the equivariance of $V$, Eqs. (1.1) and (1.2), and finally the equivariance of $\varphi$. We next differentiate Eq. (1.6) at the identity of $\mathscr{G}$. Note that $\left.\frac{d}{d t}\right|_{t=0} \operatorname{Ad}_{\exp t V} V^{\prime}$ is the group theoretical bracket $\left[V, V^{\prime}\right]_{\mathrm{grp}}$, while $\left.\frac{d}{d t}\right|_{t=0}(\exp t V)_{*} V^{\prime}=-\mathscr{L}_{V} V^{\prime}=-\left[V, V^{\prime}\right]$. Also, $A\left(\left[V, V^{\prime}\right]\right)=-\left[A(V), A\left(V^{\prime}\right)\right]$. To see this, start with $\left[V, V^{\prime}\right]=\left[V^{a} \overline{\lambda_{a}}, V^{\prime}\right]=-\left(i_{V^{\prime}} d V^{a}\right) \overline{\lambda_{a}}$, where we have used $\left[\overline{\lambda_{a}}, V^{\prime}\right]$ $=\mathscr{L}_{\overline{\lambda_{a}}} V^{\prime}=0$, a consequence of the equivariance of $V^{\prime}$ and the rigidity of $\bar{\lambda}_{a}$ (cf. Lemma A2.1 of [3]). Then $A\left(\left[V, V^{\prime}\right]\right)=-\left(i_{V^{\prime}} d V^{a}\right) \lambda_{a}=-i_{V^{\prime}} d(A(V))$, which $=-i_{V^{\prime}}\left(D_{A} A(V)-A A(V)+A(V) A\right)=-\left[A(V), A\left(V^{\prime}\right)\right]$ by the structural equation for the equivariant 0 -form $A(V)$. Hence

$$
\left[V, V^{\prime}\right]_{\mathrm{grp}}=-\left[V, V^{\prime}\right] \leftrightarrow\left[\Lambda, \Lambda^{\prime}\right] .
$$

We remark that Eqs. (1.2) and (1.7) concern vertical vector fields which are respectively rigid (but non-equivariant) and equivariant (but non-rigid).

$\mathscr{G}$ acts on $\mathscr{A}$ on the right: $A \cdot \varphi=\varphi^{*} A \leftrightarrow \gamma^{-1} A \gamma+\gamma^{-1} d \gamma$, and induces an action on its tangent vectors: $(\cdot \varphi)_{*} \tau=\varphi^{*} \tau \leftrightarrow \gamma^{-1} \tau \gamma$. The infinitesimal generators of this action are the rigid vector fields $\bar{V}$ on $\mathscr{A}$ where

$$
\bar{V}(A):=\left.\frac{d}{d t}\right|_{t=0} A \cdot(\exp t V)=\mathscr{L}_{V} A \leftrightarrow D_{A} \Lambda \quad(V \in \operatorname{Lie} \mathscr{G}) .
$$

The equivariance and horizontality of $\mathscr{L}_{V} A$ follows from the method but not the statement of Proposition A 6.1 in [3]. Also,

$$
\mathscr{L}_{V} A=i_{V}\left(F_{A}-A^{2}\right)+d\left(i_{V} A\right)=d\left(i_{V} A\right)+A i_{V} A-\left(i_{V} A\right) A \leftrightarrow D_{A} \Lambda .
$$


Each $V \in \operatorname{Lie} \mathscr{G}$ gives rise to a passive variational operator $T_{V}$ which acts on functionals of $A$ and its derivatives, such that $T_{V} A:=\bar{V}(A)=\mathscr{L}_{V} A$. For example, $T_{V} F_{A}=d \mathscr{L}_{V} A+\left(\mathscr{L}_{V} A\right) A+A \mathscr{L}_{V} A \leftrightarrow D_{A} D_{A} \Lambda=F_{A} \Lambda-\Lambda F_{A}$. The local section description of $T_{V}$ is the variational operator $T_{A}$ (such that $T_{A} A=D_{A} \Lambda$ ) in Bardeen and Zumino [4]. Next we note that if $\tau$ is a horizontal one-form, then $\mathscr{L}_{V} \tau=i_{V} d \tau$ $=i_{V}\left(D_{A} \tau-A \tau-\tau A\right)=-\left(i_{V} A\right) \tau+\tau i_{V} A \leftrightarrow \tau^{s} \Lambda-\Lambda \tau^{s}$. Hence $T_{V} T_{V^{\prime}} A=T_{V}\left(\mathscr{L}_{V^{\prime}} A\right)$ $=\mathscr{L}_{V^{\prime}}\left(T_{V} A\right)=\mathscr{L}_{V^{\prime}} \mathscr{L}_{V} A \leftrightarrow\left(D_{A} \Lambda\right) \Lambda^{\prime}-\Lambda^{\prime} D_{A} \Lambda=T_{A} T_{\Lambda^{\prime}} A$. Thus from $\mathscr{L}_{V^{\prime}} \mathscr{L}_{V}$ $-\mathscr{L}_{V} \mathscr{L}_{V^{\prime}}=\mathscr{L}_{\left[V^{\prime}, V\right]}=\mathscr{L}_{\left[V, V^{\prime}\right] \operatorname{grp}}$ and Eq. (1.7), we have

$$
\left(T_{V} T_{V^{\prime}}-T_{V^{\prime}} T_{V}\right) A=T_{\left[V, V^{\prime}\right]_{\operatorname{grp}}} A \leftrightarrow\left(T_{\Lambda} T_{\Lambda^{\prime}}-T_{\Lambda^{\prime}} T_{\Lambda}\right) A=T_{\left[\Lambda, \Lambda^{\prime}\right]} A .
$$

We remark that in comparing with Bardeen and Zumino, it is helpful to keep in mind that their Eq. (3.32), namely $T_{\Lambda} B=B \Lambda-\Lambda B$, concerns $B$ 's which are independent of $A$ and is used to simplify the bookkeeping in a subsequent calculation; this formula does not have a counterpart in the formalism we are using.

We now discuss symmetric products. Given $m g$-valued differential forms $L_{i}=L_{i}^{a} \lambda_{a}\left(\left\{\lambda_{a}\right\}\right.$ a basis for $\left.\mathfrak{g}\right)$, define the symmetric product

$$
P\left(L_{1}, \ldots, L_{m}\right):=\frac{1}{m !} \sum_{\pi \in S_{m}} L_{1}^{a_{1}} \ldots L_{m}^{a_{m}} \lambda_{\pi\left(a_{1}\right)} \ldots \lambda_{\pi\left(a_{m}\right)} .
$$

Here, $S_{m}$ is the permutation group on $m$ symbols $a_{1}, \ldots, a_{m}$. In $P(\ldots), L^{k}$ will mean $L$ repeated $k$ times. For $\tau, \xi \in \Lambda^{1} \otimes \mathfrak{g}$ and $F, K \in \Lambda^{2} \otimes \mathfrak{g}$, we have

$$
\operatorname{tr} P\left(\tau, \xi, F^{n-1}\right)=\frac{1}{n} \operatorname{tr}\left(\sum_{i=0}^{n-1} \tau F^{i} \xi F^{n-1-i}\right),
$$

and

$$
\begin{aligned}
\operatorname{tr} P\left(\tau, \xi, K, F^{n-2}\right)= & \frac{1}{n(n-1)} \operatorname{tr}\left(\tau \xi \sum_{j=0}^{n-2} F^{j} K F^{n-2-j}+\sum_{i=1}^{n-2} \tau \sum_{j=0}^{i-1} F^{j} K F^{i-1-j} \xi F^{n-1-i}\right. \\
& \left.+\sum_{i=1}^{n-2} \tau F^{i} \xi \sum_{j=0}^{n-2-i} F^{j} K F^{n-2-i-j}+\tau \sum_{j=0}^{n-2} F^{j} K F^{n-2-j} \xi\right),
\end{aligned}
$$

which shows that $\operatorname{tr} P\left(\tau, \xi, F^{n-1}\right)$ and $\operatorname{tr} P\left(\tau, \xi, K, F^{n-2}\right)$ are anti-symmetric in $\tau, \xi$. Consider for example Eq. (1.11). Among the $(n+1)$ ! elements of $S_{n+1}$, there are $n \cdot(n-1) !=n$ !, which generate $(n-1) ! \operatorname{tr}\left(\sum \ldots\right)$; each of these $n$ ! elements has $(n+1)$ cyclicly permuted companions, which all generate the same term due to the cyclicity of the trace. Thus $1 / n$ comes from $(n+1)(n-1) ! /(n+1)$ !.

Let $\mathscr{X}(\mathscr{A})$ denote the space of all vector fields on $\mathscr{A}$. To simplify later discussions we define, for each $\eta \in \mathscr{X}(\mathscr{A})$, the operator $\delta_{\eta}$ such that: $\delta_{\eta} \tau$ $=0 \forall \tau \in \mathscr{X}(\mathscr{A}), \delta_{\eta} A=\eta \forall A \in \mathscr{A}$, and $\delta_{\eta}$ acts on functionals of $\tau$ and $A$ as a variation. For example, $\delta_{\eta}\left(\tau A \xi F_{A}\right)=\tau \eta \xi F_{A}+\tau A \xi D_{A} \eta$; note however that $\delta_{\eta} F_{t A}=t D_{t A} \eta$. Thus $\delta$ is identical to the usual functional variation of $A$. From Eqs. (1.11),(1.12), we get, $\forall \tau, \xi, \eta \in \mathscr{X}(\mathscr{A})$,

$$
\delta_{\eta} \operatorname{tr} P\left(\tau, \xi, F_{t A}^{n-1}\right)=(n-1) \operatorname{tr} P\left(\tau, \xi, t D_{t A} \eta, F_{t A}^{n-2}\right) .
$$

From now on, we shall freely integrate-by-parts on $M$ and ignore boundary terms. Such is justified if suitable decay conditions are imposed on the fields, or if 
$M$ is compact without boundary. We have a Jacobi identity involving $\tau, \xi, \eta \in \Lambda^{1} \otimes \mathfrak{g}$ and $B \in \mathscr{B}$ :

$$
\int_{M^{2 n}} \operatorname{tr}\left(P\left(\xi, \eta, D_{B} \tau, F_{B}^{n-2}\right)+P\left(\eta, \tau, D_{B} \xi, F_{B}^{n-2}\right)+P\left(\tau, \xi, D_{B} \eta, F_{B}^{n-2}\right)\right)=0 .
$$

An elementary derivation begins with the identities $\int \operatorname{tr}\left(\xi \eta F^{l}(D \tau) F^{m}\right)$ $=\int \operatorname{tr}\left(-(\mathrm{D} \xi) \eta F^{l} \tau F^{m}+\xi(D \eta) F^{l} \tau F^{m}\right)$ and $\int \operatorname{tr}\left(\xi F^{l}(D \tau) F^{m} \eta F^{r}\right)=\int \operatorname{tr}\left((D \xi) F^{l} \tau F^{m} \eta F^{r}\right.$ $\left.+\xi F^{l} \tau F^{m}(D \eta) F^{r}\right)$, obtained from partial integration and the generalized Bianchi identity. Equation (1.12) and these identities convert $\operatorname{tr} P\left(\xi, \eta, D \tau, F^{n-2}\right)$ into $\operatorname{tr}\left(D \xi\right.$ terms $+D \eta$ terms), which cancel $\operatorname{tr} P\left(\eta, \tau, D \xi, F^{n-2}\right)$ and $\operatorname{tr} P\left(\tau, \xi, D \eta, F^{n-2}\right)$ respectively. We omit here the (tedious) combinatorics. A more elegant derivation may be based on Eq. (5.40) of [6].

\section{Review of Anomalies}

Let $g_{\mu \nu}$ be the components of a fixed background Riemannian metric on an evendimensional spin-manifold $M^{2 n}$. The associated volume form is $\sqrt{g} d x$, where $\sqrt{g}:=\left(\operatorname{det} g_{\mu \nu}\right)^{1 / 2}$ and $d x$ abbreviates the wedge product $d x^{1} \ldots d x^{2 n}$.

The lagrangian which describes the interaction of a Dirac spinor $\psi$ with a classical (that is, $c$-number) external non-Abelian gauge field $A$ is $L(A, \psi)=\bar{\psi} \mathscr{D}_{A} \psi$, where $\mathscr{D}_{A}$ is the Dirac operator $\gamma^{\mu}\left(\partial_{\mu}+\frac{1}{2} \omega_{\mu}^{\alpha \beta} \sigma_{\alpha \beta}+A_{\mu}\right)$ and $\omega_{\mu}^{\alpha \beta}$ are the components of the Christoffel (torsion-free) spin-connection [15]. The vacuum action functional $W(A)$ is defined through a Feynman path integral: expiW(A) $=\int_{\psi}$ meas. $(\psi) \exp \left(i \int_{M} \sqrt{g} d x L(A, \psi)\right)$. The non-invariance of $W(A)$ under the (infinitesimal) action of $\mathscr{G}$ on $\mathscr{A}$ defines, in principle, the (perturbative) nonAbelian consistent axial anomaly

$$
G(V, A):=T_{V}(W(A)), \quad V \in \operatorname{Lie} \mathscr{G} .
$$

$G$ is named consistent because, in view of Eq. (1.9), it must obey the Wess-Zumino consistency condition

$$
T_{V}\left(G\left(V^{\prime}, A\right)\right)-T_{V^{\prime}}(G(V, A))=G\left(\left[V, V^{\prime}\right]_{\mathrm{grp}}, A\right) .
$$

If the vertical equivariant vector field $V$ on $P$ is everywhere proportional to a fixed $\bar{\lambda}$, where $\lambda$ belongs to the Lie algebra of a $U(1)$ factor of the structure group, then $G(V, A)$ is called the Abelian anomaly. General arguments [17] show that the Abelian anomaly is proportional to $\int_{M} \theta \operatorname{tr} F_{A}^{n}$, where $\theta$ is a real-valued function on
$M$.

The requirements that $G(V, A)$ is linear in $V$, depends only on local data on $\mathscr{A}$, is given by the integral of a translation-invariant $2 n$-form on $M^{2 n}$, and obeys the consistency condition (2.2), are sufficient to characterize (cf. [16] and references therein) it up to redefinitions of $W(A)$ through the addition of counter-terms. An explicit formula for $G(V, A)$ has been obtained $[16,17]$ by starting with the Abelian anomaly (or more precisely the Chern character) $\operatorname{tr} F_{A}^{n+1}$ in $(2 n+2)$ dimensions and transgressing twice. The result, after suppressing a multiplicative constant $a_{n}:=i^{n+1}\left((n+1)(2 \pi)^{n+1}\right)^{-1}$, is

$$
G(V, A)=\int_{M^{2 n}}(n+1) n \int_{0}^{1} d t(1-t) \operatorname{tr}\left(\Lambda d_{M} P\left(A, F_{t A}^{n-1}\right)\right),
$$


where $d_{M}$ is the exterior derivative on $M$. Our $G$ in Eq. (2.3) is the negative of that in [17].

Formally, the non-invariance of $W(A)$ can be interpreted as the nonconservation of a certain current. Observe from Eq. (2.1) that $G(V, A)$ $=\left(d_{\mathscr{A}} W\right)(\bar{V}(A))$ and $\bar{V}(A) \leftrightarrow D_{A} \Lambda$ [Eq. (1.8)], thus

$$
G(V, A)=\int_{M^{2 n}} \operatorname{tr}\left(\sqrt{g} d x K^{\alpha}\left(D_{A} \Lambda\right)_{\alpha}\right),
$$

where $K^{\alpha}:=\left(\delta W / \delta A_{\alpha}^{b}\right) \lambda^{b}$ are the $\mathfrak{g}$-valued components of the current vector $K$. Let $[\mu \ldots \alpha]$ be the totally antisymmetric symbol on $M^{2 n}$ such that $[1 \ldots 2 n]=1$, then $\varepsilon^{\prime \mu \ldots \alpha}:=[\mu \ldots \alpha] / \sqrt{g}$ is the Levi-Civita tensor (not density). Define the $g$-valued current-(2n-1)-form $J=J_{\mu \ldots \xi} d x^{\mu} \ldots d x^{\xi}$ by the relation $\varepsilon^{\prime \mu \ldots \xi \alpha} J_{\mu \ldots \xi}=K^{\alpha}$. The right-hand side of Eq. (2.4) can then be rewritten as $\int \operatorname{tr}\left(d x^{\mu} \ldots d x^{\xi} d x^{\alpha} J_{\mu \ldots \xi}\left(D_{A} \Lambda\right)_{\alpha}\right)$ $=\int \operatorname{tr}\left(J D_{A} \Lambda\right)=\int \operatorname{tr}\left(-\left(D_{A} \Lambda\right) J\right)=\int \operatorname{tr}\left(\Lambda D_{A} J\right)$; so

$$
G(V, A)=\int_{M^{2 n}} \operatorname{tr}\left(\Lambda D_{A} J\right)=: \Lambda \cdot D_{A} J,
$$

which shows that the non-conservation of $J$ is due to $G$.

The consistent anomaly $G$ corresponds to the amplitudes of anomalous Feynman diagrams, and represents the non-conservation of certain quantum numbers. These diagrams are in the form of a fermion loop with external boson legs. If one symmetrizes the external bosons, then the resulting amplitudes correspond to the so-called covariant anomaly $\widetilde{G}$. Explicitly $[4,11-13]$

$$
\widetilde{G}(V, A)=\int_{M^{2 n}}(n+1) \operatorname{tr}\left(\Lambda F_{A}^{n}\right) .
$$

There is a current-( $2 n-1)$-form $\widetilde{J}$ such that $\Lambda \cdot D_{A} \widetilde{J}=\widetilde{G}$. It is equal to $J+X$, where [4]

$$
\eta \cdot X=\int_{M^{2 n}}(n+1) n \int_{0}^{1} t d t \operatorname{tr} P\left(\eta, A, F_{t A}^{n-1}\right)
$$

for any $\eta \in \Lambda^{1}(M) \otimes$ g. Applying $\Lambda \cdot D$ to $J+X=\widetilde{J}$ and using $\Lambda \cdot D X=-(D \Lambda) \cdot X$, we get

$$
\widetilde{G}(V, A)+\left(D_{A} \Lambda\right) \cdot X=G(V, A) .
$$

An intrinsic integrability condition for $\tilde{G}$, corresponding to the consistency condition (2.2) for $G$, will be derived in Sect. 4.

The operator $X$ also has a direct physical interpretation. In theories with Goldstone bosons and anomalies the effective action has a term

$$
\Gamma=\int_{\text {id }}^{\varphi} G
$$

which is the integrated version of Eq. (2.1). $\varphi$ is then interpreted as the Goldstone field, whose local description is $\gamma$ [see Eq. (1.4)]. From the equations of motion for $A$, one can [8] identify

$$
\operatorname{tr}\left(A d_{\varphi}^{-1} V X(A \cdot \varphi)\right) \leftrightarrow \operatorname{tr}\left(\gamma^{-1} \Lambda \gamma X\left(\gamma^{-1} A \gamma+\gamma^{-1} d \gamma\right)\right)
$$

as the covariant current of the Goldstone bosons in the direction of $V$. 
Finally, we mention a result of Atiyah and Singer [2] which relates the consistent anomaly $G$ to some elliptic differential geometric construction on $\mathscr{A}$. They consider the null space (zero-frequency modes) of $\mathscr{D}_{A} \circ\left(1+\gamma_{2 n+1}\right) / 2$, and that of its adjoint, as fibers over each $A \in \mathscr{A}$. Taking the formal difference (as in $K$-theory) of these fibers, one eliminates the jumps in dimension as $A$ varies, and obtains a vector bundle over $\mathscr{A}$, the index bundle. Since the affine space $\mathscr{A}$ is topologically trivial, the first Chern form $c_{1}$ of the index bundle, being a closed 2 -form on $\mathscr{A}$, is globally exact. Hence $c_{1}=d_{\mathscr{A}} \beta$ for some one-form $\beta$ on $\mathscr{A}$. It is a corollary of their general constructions that (again we suppress the aforementioned constants $a_{n}$ )

$$
\beta(\bar{V}(A))=\int_{M^{2 n}}(n+1) s^{*}\left(i_{V} \int_{0}^{1} d t \operatorname{tr}\left(A F_{t A}^{n}\right)\right)=G(V, A) .
$$

Here, $s: U \subseteq M \rightarrow P$ is a local section, $F_{t A}$ stands for the 2-form $t d A+t^{2} A^{2}=t F_{A}$ $+\left(t^{2}-t\right) A^{2}$ on $P$, and $\int_{0}^{1} d t \operatorname{tr}\left(A F_{t A}^{n}\right)$ is a Chern-Simons secondary invariant [6]. The fact that the integral in Eq. (2.10) equals $G$ can be verified by using the identity $s^{*}\left(i_{V} \operatorname{tr}\left(A F_{t A}^{n}\right)\right)=\operatorname{tr}\left(\Lambda F_{t A}^{n}\right)+n\left(t^{2}-t\right) \operatorname{tr} P\left(A, A \Lambda-\Lambda A, F_{t A}^{n-1}\right)$, followed by Eqs. (B-27) $\rightarrow(\mathrm{B}-33)$ of $[17]$.

In the next section we will study the covariant anomaly $\tilde{G}$ from the viewpoint of the presymplectic geometry on $\mathscr{A}$.

\section{Main Results}

In this section, $d$ denotes the exterior derivative on $\mathscr{A}$. If $\Phi$ is an $r$-form on $\mathscr{A}, i_{\tau} \Phi$ will sometimes be used to denote the $(r-1)$-form $\Phi(\tau, \ldots)$. Let $[\tau, \xi]$ be the Lie bracket for vector fields on $\mathscr{A}$. We abbreviate the expression $\left(\tau_{\alpha}^{c}(A) \partial \xi_{\mu}^{a}(A) / \partial A_{\alpha}^{c}\right) \partial / \partial A_{\mu}^{a}$ by $i_{\tau} d \xi$, so that $[\tau, \xi]=i_{\tau} d \xi-i_{\xi} d \tau$. We also recall the operators $T_{V}$ and $\delta_{\tau}$ from Sect. 1 .

If $\alpha$ is a one-form on $\mathscr{A},(d \alpha)(\tau, \xi)=i_{\tau} d(\alpha(\xi))-i_{\xi} d(\alpha(\tau))-\alpha([\tau, \xi])=\delta_{\tau}(\alpha(\xi))$ $+\alpha\left(i_{\tau} d \xi\right)-\delta_{\xi}(\alpha(\tau))-\alpha\left(i_{\xi} d \tau\right)-\alpha([\tau, \xi])$. Thus

$$
(d \alpha)(\tau, \xi)=\delta_{\tau}(\alpha(\xi))-\delta_{\xi}(\alpha(\tau)) .
$$

Likewise, if $\omega$ is a 2 -form on $\mathscr{A}$, then from $(d \omega)(\tau, \xi, \eta)=i_{\tau} d(\omega(\xi, \eta))-i_{\xi} d(\omega(\tau, \eta))$ $+i_{\eta} d(\omega(\tau, \xi))-\omega([\tau, \xi], \eta)+\omega([\tau, \eta], \xi)-\omega([\xi, \eta], \tau)$, we get

$$
(d \omega)(\tau, \xi, \eta)=\delta_{\tau}(\omega(\xi, \eta))+\delta_{\xi}(\omega(\eta, \tau))+\delta_{\eta}(\omega(\tau, \xi)) .
$$

For now, let $\omega$ be an arbitrary but fixed closed 2-form on $\mathscr{A}$. Since $\mathscr{A}$ is affine and hence topologically trivial, $\omega$ is globally exact. Let $\alpha$ be any globally defined one-form on $\mathscr{A}$ such that $d \alpha=\omega$. (We will soon specialize to a preferred $\omega$ and $\alpha$.) Let $\tilde{H}:(\operatorname{Lie} \mathscr{G}) \times \mathscr{A} \rightarrow \mathbb{R}$ be any function which is linear in Lie $\mathscr{G}$; motivated by Eq. (2.8), we define its transform $H:(\operatorname{Lie} \mathscr{G}) \times \mathscr{A} \rightarrow \mathbb{R}$ (also linear in Lie $\mathscr{G}$ ) by

$$
H(V, A):=\tilde{H}(V, A)+\alpha(\bar{V}(A)),
$$

that is, $H(\Lambda, A):=\tilde{H}(\Lambda, A)+\alpha\left(D_{A} \Lambda\right)$. Using the fact that $T_{V}\left(\alpha\left(\bar{V}^{\prime}(A)\right)\right)$ $=\delta_{\bar{V}}\left(\alpha\left(\bar{V}^{\prime}(A)\right)\right)+\alpha\left(T_{V} T_{V^{\prime}} A\right)$, Eqs. (1.9) and (3.1), and $d \alpha=\omega$, we see that $H$ satisfies the Wess-Zumino consistency condition

$$
T_{V}\left(H\left(V^{\prime}, A\right)\right)-T_{V^{\prime}}(H(V, A))=H\left(\left[V, V^{\prime}\right]_{\mathrm{grp}}, A\right)
$$


iff $\tilde{H}$ satisfies

$$
T_{V}\left(\tilde{H}\left(V^{\prime}, A\right)\right)-T_{V^{\prime}}(\tilde{H}(V, A))+\omega\left(\bar{V}(A), \overline{V^{\prime}}(A)\right)=\tilde{H}\left(\left[V, V^{\prime}\right]_{\mathrm{grp}}, A\right) .
$$

We next require that $\omega$ be gauge-invariant, that is, $(\cdot \varphi)^{*} \omega=\omega$. A function $\tilde{H}:($ Lie $\mathscr{G}) \times \mathscr{A} \rightarrow \mathbb{R}$ is said to be a momentum mapping [1] for the action of $\mathscr{G}$ on $(\mathscr{A}, \omega)$ if it is linear in Lie $\mathscr{G}$ and

$$
d(\tilde{H}(V, \cdot))=\omega(\bar{V}, \cdot), \quad \text { that is, } \quad \delta_{\xi}(\tilde{H}(V, A))=\omega(\bar{V}(A), \xi) .
$$

In other words, $\tilde{H}$ is a momentum mapping if, with respect to the presymplectic structure $\omega, \bar{V}$ is the hamiltonian vector field of the function $\tilde{H}(V, \cdot)$ on $\mathscr{A}$. The momentum mapping $\tilde{H}$ is said to be equivariant if

$$
\tilde{H}\left(V^{\prime}, A \cdot \varphi\right)=\tilde{H}\left(\operatorname{Ad}_{\varphi} V^{\prime}, A\right) .
$$

Differentiating this (with the chain rule) at the identity of $\mathscr{G}$, and using Eq. (3.6), we get

$$
-\omega\left(\bar{V}(A), \overline{V^{\prime}}(A)\right)=\tilde{H}\left(\left[V, V^{\prime}\right]_{\mathrm{grp}}, A\right),
$$

that is, $-\omega\left(D_{A} \Lambda, D_{A} \Lambda^{\prime}\right)=\tilde{H}([\Lambda, \Lambda], A)$. Equation (3.8) is the criterion for infinitesimal equivariance. Our definition of equivariance is a slight modification of that in [1], which treats left actions. From the same reference, one learns that the cohomology class of $\mathscr{G}$ defined by an equivariant momentum mapping is trivial. Next note that since $\mathscr{A}$ is path-connected $\left[A+t\left(A^{\prime}-A\right)\right.$ is a ray in $\mathscr{A}$ from any $A$ to any $A]$ and hence connected, Eq. (3.6) says that any two momentum mappings must differ by a function $C(V)$ which is independent of $A$ and linear in $V$. From Eq. (3.8) we see that the additional requirement of infinitesimal equivariance on the two momentum mappings translates into the restriction that $C\left(\left[V, V^{\prime}\right]_{\text {grp }}\right)$ must vanish for any $V, V^{\prime} \in \operatorname{Lie} \mathscr{G}$.

Let us restrict to the class of H's for which $\tilde{H}$ is a momentum mapping. Then $H$ satisfies the Wess-Zumino consistency condition (3.4) iff $\tilde{H}$ is an infinitesimally equivariant momentum mapping. The reason being that in such case, $T_{V}\left(\tilde{H}\left(V^{\prime}, A\right)\right)$ $=\delta_{\bar{V}}\left(\tilde{H}\left(V^{\prime}, A\right)\right)=\omega\left(\overline{V^{\prime}}(A), \bar{V}(A)\right)$, and thus the left-hand side of Eq. (3.5) simplifies to $-\omega\left(\bar{V}(A), \overline{V^{\prime}}(A)\right)$.

We now specialize to the following closed $\mathscr{G}$-invariant 2 -form on $\mathscr{A}$ :

$$
\begin{aligned}
\omega_{A}(\tau, \xi): & =-\int_{M^{2 n}}(n+1) \operatorname{tr}\left(\sum_{i=0}^{n-1} \tau F_{A}^{i} \xi F_{A}^{n-1-i}\right) \\
& =-\int_{M^{2 n}}(n+1) n \operatorname{tr} P\left(\tau, \xi, F_{A}^{n-1}\right) .
\end{aligned}
$$

The definition is clearly independent of the choice of local sections. Equation (1.11) shows that $\omega$ is skew and can be written in the above two ways. It is closed because of Eqs. (3.2), (1.13), and the Jacobi identity (1.14). It is $\mathscr{G}$-invariant: $\left[(\cdot \varphi)^{*} \omega\right]_{A}(\tau, \xi)$ $=\omega_{A \cdot \varphi}\left((\cdot \varphi)_{*} \tau,(\cdot \varphi)_{*} \xi\right)=-\int(n+1) n \operatorname{tr} P\left(\gamma^{-1} \tau \gamma, \quad \gamma^{-1} \xi \gamma, \quad\left[\gamma^{-1} F_{A} \gamma\right]^{n-1}\right)=\omega_{A}(\tau, \xi)$. We believe that, up to a constant multiple, this is the only 2 -form on $\mathscr{A}$ which is closed, $\mathscr{G}$-invariant, with values depending only on local data on $\mathscr{A}$, and is given by the integral of a translation-invariant section-independent $2 n$-form on $M^{2 n}$.

Consider Eqs. (3.6) and (3.8) with this $\omega$, and the identity

$$
-\int \operatorname{tr}\left((D \Lambda) F^{l} \xi F^{m}\right)=\int \operatorname{tr}\left(\Lambda F^{l}(D \xi) F^{m}\right) .
$$


We have

$$
\begin{aligned}
\omega(\bar{V}(A), \xi) & =\int(n+1) \operatorname{tr}\left(\Lambda \sum_{i=0}^{n-1} F_{A}^{i}\left(D_{A} \xi\right) F_{A}^{n-1-i}\right) \\
& =\int(n+1) \operatorname{tr}\left(\Lambda \delta_{\xi} F_{A}^{n}\right)=\delta_{\xi}(\widetilde{G}(V, A))
\end{aligned}
$$

and

$$
\begin{aligned}
-\omega\left(\bar{V}(A), \overline{V^{\prime}}(A)\right) & =-\int(n+1) \operatorname{tr}\left(\sum_{i=0}^{n-1} \Lambda F_{A}^{i}\left(F_{A} \Lambda^{\prime}-\Lambda^{\prime} F_{A}\right) F_{A}^{n-1-i}\right) \\
& =\int(n+1) \operatorname{tr}\left(\left[\Lambda, \Lambda^{\prime}\right] F_{A}^{n}\right)=\widetilde{G}\left(\left[V, V^{\prime}\right]_{\mathrm{grp}}, A\right) .
\end{aligned}
$$

Thus all infinitesimally equivariant momentum mappings are of the form $\tilde{H}(V, A)$ $=\widetilde{G}(V, A)+C(V)$, where $C(V)$ is any linear function on Lie $\mathscr{G}$ (and independent of $A$ ) which vanishes on brackets. $\widetilde{G}$ is actually equivariant (not just infinitesimally so) since $\operatorname{tr}\left(\Lambda\left(\gamma^{-1} F_{A} \gamma\right)^{n}\right)=\operatorname{tr}\left(\gamma \Lambda \gamma^{-1} F_{A}^{n}\right)$; we emphasize that the equivariance of $\widetilde{G}$ is to be distinguished from its section-independence. The $C$ 's are of limited significance here because if one insists that $\tilde{H}(V, A)$, and hence $C(V)$, is given by the integral over $M^{2 n}$ of a quantity which obeys locality, then [14] for each choice of $C$, counter-terms can be added to the action functional so that $\widetilde{G}+C$, rather than $\widetilde{G}$, is the covariant anomaly.

Let us determine $\alpha$ up to closed one-forms. Since $\mathscr{A}$ is affine and hence starshaped about any fixed element $A_{0}$, there is a linear map [10] $I$ from 2-forms to one-forms such that $I d+d I=$ the identity map. Explicitly,

$$
(I \omega)_{A}(\eta):=\int_{0}^{1} t d t \omega_{A_{t}}\left(\eta, A_{0}-A\right)=\int_{M^{2 n}}(n+1) n \int_{0}^{1} t d t \operatorname{tr} P\left(\eta,-\left(A_{0}-A\right), F_{A_{t}}^{n-1}\right),
$$

where $A_{t}:=A_{0}+t\left(A-A_{0}\right)$ is the ray in $\mathscr{A}$ from $A_{0}$ to $A$ and $F_{A_{t}}=F_{t A}+F_{(1-t) A_{0}}$ $+\left(t-t^{2}\right)\left(A A_{0}+A_{0} A\right)$ is the curvature of the connection $A_{t}$. The tangent vectors $\eta$ and $A_{0}-A$, though based at the point $A$, are equivariant horizontal $g$-valued oneforms on $P$ and, since the notion of horizontal forms is independent of any connection, we can just as well regard them as tangent vectors based at $A_{t}$. Note that $I \omega$ is independent of local sections, though it is not $\mathscr{G}$-invariant: $\left((\cdot \varphi)^{*}(I \omega)\right)_{A}(\eta)=(I \omega)_{A \cdot \varphi}\left((\cdot \varphi)_{*} \eta\right)=\int_{0}^{1} t d t \omega_{(A \cdot \varphi)_{t}}\left((\cdot \varphi)_{*} \eta, A_{0}-A \cdot \varphi\right) \neq(I \omega)_{A}(\eta)$.

From the homotopy property of $I$ and the closure of $\omega$, we have $d(I \omega)=\omega$. Thus $I \omega$ is one choice for $\alpha$. Observe that $I \omega$ is the sum of two parts, one which is homogeneous in $A_{0}$, and one which is independent of $A_{0}$. We now show that the latter part is also an acceptable choice for $\alpha$. Let

$$
\alpha_{A}(\eta):=\int_{M^{2 n}}(n+1) n \int_{0}^{1} t d t \operatorname{tr} P\left(\eta, A, F_{t A}^{n-1}\right) .
$$

Since there is no zero connection, this $\alpha$ is not obtainable from $I \omega$ by choosing any $A_{0}$; we must therefore explicitly check that $d \alpha=\omega$. Using Eq. (3.1), a variant of Eq. (1.13), and $\delta_{\eta} F_{t A}=t D_{t A} \eta$, we have

$$
\begin{aligned}
(d \alpha)_{A}(\tau, \xi)= & \int_{M^{2 n}}(n+1) n \int_{0}^{1} d t \operatorname{tr}\left(P\left(\xi, t \tau, F_{t A}^{n-1}\right)+(n-1) P\left(\xi, t A, t D_{t A} \tau, F_{t A}^{n-2}\right)\right. \\
& \left.-P\left(\tau, t \xi, F_{t A}^{n-1}\right)-(n-1) P\left(\tau, t A, t D_{t A} \xi, F_{t A}^{n-2}\right)\right)
\end{aligned}
$$


which, upon the use of the antisymmetry in the first two slots of these tr $P$ 's, and the Jacobi identity (1.14), becomes

$$
\begin{aligned}
= & -\int_{M^{2 n}}(n+1) n \int_{0}^{1} d t \operatorname{tr}\left(P\left(\tau, t \xi, F_{t A}^{n-1}\right)+P\left(t \tau, \xi, F_{t A}^{n-1}\right)\right. \\
& \left.+(n-1) P\left(\tau, \xi, t D_{t A} t A, F_{t A}^{n-2}\right)\right) .
\end{aligned}
$$

Rewriting the third integrand as

$$
\operatorname{tr}(n-1) P\left(\tau, \xi, t^{2}(d / d t) F_{t A}, F_{t A}^{n-2}\right)=\operatorname{tr}(n-1) P\left(t \tau, t \xi,(d / d t) F_{t A}, F_{t A}^{n-2}\right),
$$

and using a variant of Eq. (1.13), the above integral

$$
=-\int_{M^{2 n}}(n+1) n \int_{0}^{1} d t \frac{d}{d t} \operatorname{tr} P\left(t \tau, t \xi, F_{t A}^{n-1}\right)=-\int_{M^{2 n}}(n+1) n \operatorname{tr} P\left(\tau, \xi, F_{A}^{n-1}\right)=\omega_{A}(\tau, \xi) .
$$

This one-form $\alpha$ on $\mathscr{A}$ is neither section-independent nor $\mathscr{G}$-invariant, and its integral representation on $M^{2 n}$ is given by the $X$ operator of Bardeen and Zumino [4], in the sense that $\alpha(\xi)=\xi \cdot X$. Corresponding to the choice $\tilde{H}=\widetilde{G}$, the solution $H$ of the Wess-Zumino consistency condition (3.4) is $\widetilde{G}(V, A)+\alpha(\bar{V}(A))=\widetilde{G}(\Lambda, A)$ $+\left(D_{A} \Lambda\right) \cdot X$ which, in view of Eq. (2.8), is the consistent anomaly $G$. From the paragraph following Eq. (3.8), one sees that the infinitesimal equivariance of $\widetilde{G}$ is equivalent to the Wess-Zumino consistency of $G$. We summarize,

Proposition. Let $\omega$ be the closed $\mathscr{G}$-invariant 2-form on $\mathscr{A}$ given by Eq. (3.9).

A. Then, up to linear functions on Lie $\mathscr{G}$ which vanish on brackets,

(i) The covariant anomaly $\tilde{G}$ is the unique infinitesimally equivariant momentum mapping for the action of $\mathscr{G}$ on $(\mathscr{A}, \omega)$. It is also equivariant.

(ii) For any one-form $\alpha$ on $\mathscr{A}$ such that $d \alpha=\omega, \widetilde{H}=\widetilde{G}$ is the only choice of momentum mapping for which $H(V, A):=\tilde{H}(V, A)+\alpha(\bar{V}(A))$, that is $H(\Lambda, A):=\tilde{H}(\Lambda, A)+\alpha\left(D_{A} \Lambda\right)$, satisfies the Wess-Zumino consistency condition.

B. If $\alpha$ (such that $d \alpha=\omega)$ is further specified by Eq. (3.11), then $\alpha(\xi)=\xi \cdot X$ and $\tilde{G}(V, A)+\alpha(\bar{V}(A))$ is the consistent anomaly $G$. Furthermore, the infinitesimal equivariance of $\widetilde{G}$ is equivalent to the Wess-Zumino consistency of $G$.

\section{Discussion}

Some questions raised by our approach are

A. The Degeneracy of $\omega$. Being a presymplectic structure on an infinite dimensional space, the relevant notion for $\omega$ is that of weak non-degeneracy: Does $\omega(\tau, \xi)=0 \forall \xi \Rightarrow \tau=0$ ? From Eq. (3.9) we see that $\omega$ is weakly non-degenerate only when $\operatorname{dim} M=2$, in which case it gives a symplectic structure on $\mathscr{A}$. In higher dimensions (of $M$ ), its defining expression is homogeneous in $F$, and thus vanishes identically at flat connections; rewriting $\omega_{A}(\tau, \xi)$ as $-\int \operatorname{tr}\left(\left[\sum_{i=0}^{n-1} F_{A}^{n-1-i} \tau F_{A}^{i}\right] \xi\right)$, one sees that whenever the structure group is compact semi-simple, the degenerate directions $\tau$ at each $A$ are characterized by the equation $\operatorname{tr}\left(\left[\sum_{i=0}^{n-1} F_{A}^{n-1-i} \tau F_{A}^{i}\right] \lambda^{a}\right) \lambda_{a}=0$, whose left-hand side is to be understood as the 
projection onto $g$ of a matrix-valued $(2 n-1)$-form on $M^{2 n}$. The fact that for certain choices of $P$ [for example: $\operatorname{dim} M=4$ and all compact semi-simple structure groups except $\mathrm{SU}(N \geqq 3)$ and $\mathrm{SO}(6)]$ the theory is anomaly-free, certainly suggests that part of the degeneracy of $\omega$ is of group theoretical origin.

Since $\omega$ is gauge-invariant, one can also address the issue of its weak nondegeneracy on $\mathscr{A} / \mathscr{G}^{\prime}$, where $\mathscr{G}^{\prime}$ consists of those gauge transformations on $P$ which equal the identity on a certain fixed fibre in $P$. Unlike $\mathscr{G}$, the group $\mathscr{G}^{\prime}$ does act without fixed points on $\mathscr{A}$, thereby ensuring that $\mathscr{A} / \mathscr{G}^{\prime}$ is a manifold.

B. The Level Sets of $\tilde{G}$. Here we find it convenient to view the equivariant momentum mapping $\widetilde{G}$ as a map from $\mathscr{A}$ into $\left(\operatorname{Lie}^{G}\right)^{*}$, the dual space of Lie $\mathscr{G}$; and $\widetilde{G}(V, A)$ will be written as $\widetilde{G}_{A}(V)$. This view explains the nomenclature since linear and angular momenta are maps from the cotangent bundle $[(q, p)$-space $]$ of Euclidean space into the dual of the Lie algebra of the translation and rotation groups, respectively (cf. [1]).

Note that Lie $\mathscr{G}$ inherits from $g$ an Ad-invariant [by Eq. (1.6)] and sectionindependent [by Eq. (1.5)] inner product $\left(V, V^{\prime}\right):=\int_{M} \sqrt{g} d x \operatorname{tr}\left(\Lambda \Lambda^{\prime}\right)$. Thus, to $\tilde{G}$ we can associate a map $\tilde{G}^{\#}: \mathscr{A} \rightarrow \operatorname{Lie} \mathscr{G}$, defined by $\left(\tilde{G}^{\#}, V^{\prime}\right)=\widetilde{G}\left(V^{\prime}\right)$; for each $A \in \mathscr{A}$, the element $\widetilde{G}_{A}^{\#}$ in Lie $\mathscr{G}$, which is an equivariant vertical vector field on $P$, can be described via local sections as $\operatorname{tr}\left(\varepsilon^{\prime \mu \alpha \alpha}\left(F_{A}^{n}\right)_{\mu \ldots \alpha} \lambda^{a}\right) \lambda_{a}$, where $\varepsilon^{\prime}$ is the Levi-Civita tensor defined in Sect. 2. The norm square of $\tilde{G}$ can then be defined as $\|\tilde{G}\|^{2}:=\left(\widetilde{G}^{\#}, \widetilde{G}^{\#}\right)=\tilde{G}\left(\widetilde{G}^{\#}\right)$, which is a real-valued function on $\mathscr{A}$. One can ask what the level sets of $\|\widetilde{G}\|^{2}$ (henceforth abbreviated as $f$ ) look like, and whether they have any physical significance.

One can investigate the level sets of $f$ with Morse theory, the use of which on functions constructed out of momentum mappings is not new in pure mathematics (cf. [9] and references therein) nor in mathematical relativity [7]. In our case, a scheme goes as follows. The function $f$ is expected to have degenerate critical points (those where the Hessian is not an isomorphism). These degeneracies are hopefully milder for $\bar{f}$, the restriction of $f$ to a suitable submanifold of $\mathscr{A}$. A generalized Morse lemma is applied to $\bar{f}$. Findings about $\bar{f}$ are translated into those about $f$ by using an appropriate slice theorem for the action of $\mathscr{G}$ on $\mathscr{A}$, and exploiting the equivariance of $\widetilde{G}$. We anticipate the degeneracy of $\omega$ to contribute non-trivial difficulties towards the implementation of the aforementioned program.

Acknowledgements. We thank Jerry Marsden and Richard Montgomery for discussions about the concept of momentum mappings in mechanics, and Phil Yasskin for clarifications on Eq. (1.7).

\section{References}

1. Abraham, R., Marsden, J.E.: Foundations of mechanics. 2nd ed. Reading, Mass.: Benjamin/Cummings 1978

2. Atiyah, M.F., Singer, I.M.: Dirac operators coupled to vector potentials. Proc. Natl. Acad. Sci. USA 81, 2597-2600 (1984)

3. Bao, D., Isenberg, J., Yasskin, P.: The dynamics of the Einstein-Dirac system I: A principal bundle formulation of the theory and its canonical analysis. Ann. Phys. 163 (1985) 
4. Bardeen, W.A., Zumino, B.: Consistent and covariant anomalies in gauge and gravitational theories. Nucl. Phys. B 244, 421-453 (1984)

5. Bishop, R.L., Crittenden, R.J.: Geometry of manifolds. New York, London: Academic Press 1964

6. Chern, S.S.: Complex manifolds without potential theory (with an appendix on the geometry of characteristic classes). 2nd ed. Berlin, Heidelberg, New York: Springer 1979

7. Fischer, A., Marsden, J.E., Moncrief, V.: The structure of the space of solutions of Einstein's equations. I. One Killing field. Ann. Inst. H. Poincaré 33, 147-194 (1980)

8. Karabali, D., Nair, V.P., Rodgers, V.G.J.: Anomalous symmetries and induced currents. IAS Princeton, preprint (December 1984)

9. Kirwan, F.C.: Cohomology of quotients in symplectic and algebraic geometry. Mathematical notes \#31. Princeton, NJ: Princeton University Press 1984

10. Lang, S.: Differential manifolds. Reading, Mass.: Addison-Wesley 1972

11. Niemi, A.J., Semenoff, G.: Axial anomaly induced fermion fractionization and effective gauge theory actions in odd dimensional spacetimes. Phys. Rev. Lett. 51, 2077 (1983)

12. Paranjape, M.B.: Thesis, M.I.T. (1984)

13. Paranjape, M.B.: Some aspects of anomalies of global currents. Phys. Lett. 156 B, 376 (1985)

14. Piguet, O., Rouet, A.: Symmetries in perturbative quantum field theory. Phys. Rep. 76 C, 1-77 (1981)

15. Weinberg, S.: Gravitation and cosmology: Principles and applications of the general theory of relativity. New York: Wiley 1972

16. Zumino, B.: Chiral anomalies and differential geometry. In: Les Houches Lectures August 1983. DeWitt, B., Stora, R. (eds.), Amsterdam: North-Holland 1984

17. Zumino, B., Wu, Y.S., Zee, A.: Chiral anomalies, higher dimensions, and differential geometry. Nucl. Phys. B 239, 477-507 (1984)

Communicated by S.-T. Yau

Received April 26, 1985

Note added on proof. In the above expression for $\widetilde{G}^{\sharp}$, one can lower the indices on the Levi-Civita tensor and raise the indices on $F^{n}$. It is then easy to see that in $2 n$ dimensions, the norm square of $\tilde{G}$ is proportional to the norm square of the Lie algebra valued part of the matrix valued form $F^{n}$, and is therefore proportional to the Yang-Mills action functional only when $n=1$. In the $n=1$ case, our presymplectic structure is symplectic and agrees with that used by Goldman, W.: The symplectic nature of fundamental groups of surfaces. Adv. Math. 54, 200-225 (1984). Also, in the $n=1$ case, our momentum mapping is implicit in Atiyah,M.F., Bott, R.: The Yang-Mills equations over Riemann surfaces. Phil. Trans. R. Soc. Lond. A 308, 523-615 (1982). These references are brought to our attention, respectively, by J.Marsden and K. Uhlenbeck. 